\title{
Hyperthermia: Clinical Applications and Theoretical Models
}

\author{
Habib E. Ashoor \\ Department of Physics, College of Science, University of Bahrain, Sakhir, Kingdom of Bahrain \\ Email: hashoor@uob.edu.bh
}

How to cite this paper: Ashoor, H.E. (2022) Hyperthermia: Clinical Applications and Theoretical Models. Journal of Biosciences and Medicines, 10, 56-71. https://doi.org/10.4236/jbm.2022.103007

Received: January 17, 2022

Accepted: March 5, 2022

Published: March 8, 2022

Copyright (c) 2022 by author(s) and Scientific Research Publishing Inc. This work is licensed under the Creative Commons Attribution International License (CC BY 4.0).

http://creativecommons.org/licenses/by/4.0/

\begin{abstract}
Nanotechnology applications, which aid radiotherapy and chemotherapy, have revolutionized diagnosis, treatment planning, imaging, and medical machinery. The ability of nanoparticles to kill or freeze cancer cells by raising heat locally is a prominent application of nanoparticles. This paper will review the research on clinical applications of hyperthermia using nanoparticles stimulated by an alternating magnetic field and electromagnetic waves such as microwave and laser, as well as the foundation of the theoretical model used in bio-thermal applications.
\end{abstract}

\section{Keywords}

Hyperthermia, Nanoparticles, Nanotechnology, Bio-Heat Equation, Plasmon, Local Heat

\section{Introduction}

A growing body of literature has investigated heat as a therapeutic by ablating cancer cells using lethal levels of heat that irreparably coagulate proteins and other biological molecules and cause cell death. Hyperthermia (HT) is a milder increase in temperature between 41 and 50 degrees Celsius that does not cause death but does limit some collateral damage to normal tissue and can be used as a therapeutic method [1] [2] [3]. There are three ways to use hyperthermia as a treatment mechanism: local, regional, and whole body. Local hyperthermia is reserved for tumors measuring 3 to 6 centimetres in diameter [4]. Regional hyperthermia is commonly used to treat large tissue areas by using an external source of heat to raise the temperature of an organ or limb [5]. Whole-body hyperthermia can be used to treat metastatic cancerous cells that have spread throughout the body [5]. It is worth noting that a number of parameters influence increasing the temperature within the targeted tissues, such as temperature 
homogeneity in the targeted tissue, tissue type, and treatment context. This increase in temperature in a targeted tissue causes verification of vascular permeability, elevation of blood flow, and thus tumor oxygenation. Eventually, hyperthermia reduces tissue hypoxia and can be used in conjunction with radiation or anti-cancer drugs to boost their cytotoxic effects on the tumor [6] [7] [8] [9] [10].

There are numerous challenges that arise when using hyperthermia to target tumoral cells while not affecting normal cells. For example, conventional heat delivery methods, such as microwave, radiofrequency, laser, and ultrasound, are ineffective because the majority of the heat is dissipated in healthy cells located along the path of the external beam of radiation and surrounding cells. As a result, patients may experience serious side effects [11]. The ineffectiveness of conventional approaches motivates researchers to investigate an alternative hyperthermia approach. This method is based on the use of nanoparticles because hyperthermia mediators involve raising the temperature of a specific region of the body above the threshold temperature, which is $41^{\circ} \mathrm{C}$.

Nanotechnology has instilled a new spirit in the field of medicine, particularly in diagnostics and treatments [12] [13] [14]. Nanoparticles can be used to kill cancerous cells while leaving healthy cells alone. There are numerous challenges when using hyperthermia to target tumoral cells while leaving normal cells alone. Furthermore, the interactions of some nanoparticles with their biological environment, and thus their toxicity, vary with morphology, size, and surface modifications such as the addition of biocompatible coatings and targeting moieties, as well as the route of administration; therefore, each formulation must be thoroughly tested in vitro and in vivo. Magnetic Iron Oxide Nanoparticles, for example, can mediate toxicity via a variety of mechanisms, all of which must be considered when assessing their safety. The generation of reactive oxygen species causes the majority of intracellular toxicity, whereas in vivo disturbances of food clotting, iron homeostasis, and macrophage function, as well as organ toxicities, are also taken into account [15]. Traditional heat delivery methods, for example, microwave, radiofrequency, laser, and ultrasound, are ineffective because the majority of the heat is dissipated in healthy cells along the path of the external beam of radiation and surrounding cells. As a result, patients may suffer from severe side effects cells. Moreover, most studies utilize nanoparticle applications with other therapies taking advantage of the synergistic effects between them, including chemotherapy, gene therapy, immunotherapy, photodynamic therapy and radiotherapy [16]. In this review, the widespread applications and theoretical models used in nanoparticle hyperthermia will be summarized.

\section{Traditional Hyperthermia}

Various clinical methods for hyperthermia have been used, including electro- 
magnetic radiation such as microwave, laser, and radiofrequency or high intensity focused ultrasound. In the application of heat exposure in hyperthermia. As previously stated, there are three major types based on the patient's body context: local, regional, and whole [17]. Local treatment, which is devoted to a relatively small cancerous area within the targeted tissues ( $3 \mathrm{~cm}$ up to $5-6$ in diameters), can be accomplished by using customized probes and applicators placed close to the tumor independently. A grid can also be used to provide relatively homogeneous heat to the tumor [18]. While this method is recommended for reducing unwanted side effects, it is only applicable to superficial or accessible cavities in the human body, such as the rectum or the oesophagus. Regional or sectional-body hyperthermia refers to the heating of large sections of the body, such as the abdominal cavity, a specific organ, or a limb. This can be accomplished by combining a heated solution of anticancer agents with a patient's warmed blood, where the profusion of tumor-bearing limb peritoneal malignancies such as mesothelioma is performed [19]. It should be noted that this method is difficult to repeat with a reproducible heating level. Furthermore, it is commonly used to treat cancerous cells that have progressed in the major or minor pelvis and abdomen, including cervical, proctate, bladder, colorictical, and ovarian sarcomas [20]. Whole body thermia is a treatment that uses hot blankets and a thermal water path to destroy metastatic disease such as melanoma, soft tissue sarcoma, or overian cancer. Although whole-body thermia maintains a constant temperature throughout the body, it can cause complications such as thermal stress to the heart, lungs, liver, and brain [21] [22]. Luminal and interstitial hyperthermia techniques, on the other hand, imply that the source is externally placed or that customized probes are used near the tumor. As a result, challenges arise when using traditional technologies [23]. Furthermore, non-invasive ultrasound and elactromagnetic procedures have a short outcome. For example, it is difficult to reach a deep tumor, which frequently results in inhomogeneous heat delivery; additionally, heat in its flow towards the tumor may cause unwanted heat, which may damage normal cells in close proximity [24]. Radiotherapy, on the other hand, is an effective technique for treating cancer; however, severe side effects are caused by the toxicity of the radiosensitization agent administered to a patient [25]. An alternative technique that could overcome the limitations of traditional hyperthermia should be tumor-focused, less invasive, and incorporate greater homogeneity of local heat distribution, which could be accomplished by leveraging the properties of nanoparticles.

\section{Nanoparticle and Tumor Ablation}

Nanoparticles with dimensions ranging from $100 \mathrm{~nm}$ to 1 micron can be used to target cancerous cells, according to the definition. They can be stimulated by radiation, photonic, thermal, or alternating magnetic fields to raise the temperature to specific levels in order to ablate cancer cells. While radiotherapy has significant toxicity, hyperthermia-using nanoparticles improve tumor theranos- 
tics while avoiding cytotoxic effects on healthy cells. In addition, nanoparticles enable the localization of induced hyperthermia [26] [27]. There are three streams based on the method used to stimulate the heat generated by the nanoparticles: photo-thermal, magneto-thermal, and radio-thermal.

In theory, nanomedicine is the use of nanoparticles to specifically target tumor cells or immune cells of interest. It may also be capable of multiplexing. It is used in imaging, diagnostics, and therapeutics, among other things. Nanoparticles are superior photon-to-heat conversion transducers for photothermal therapy due to their tip-enhanced plasmonics and tunable plasmonic absorption in the near-infrared tissue optical window. Nanomedicine provides advantages such as increased chemotherapeutic solubility, drug encapsulation, and improved biodistribution, and it can ensure tumor-directed drug delivery and release through passive and active targeting approaches. Plasmonic (noble metal) nanoparticles, on the other hand, have tunable electronic and optical properties, allowing them to be used in cancer photothermolysis using laser nanoparticles [28] [29] [30].

Better drug targeting and delivery, lower toxicity while maintaining therapeutic effects (Therapeutic Index; TI), increased safety and biocompatibility, and faster development of new safe medicines are the primary goals of using nanomaterials in medicine. The goal of drug nanoparticle entrapment and related pharmaceutical development, on the other hand, is to improve drug delivery or uptake by target cells and/or to reduce the toxicity of the free drug to non-target organs. Both scenarios will increase the therapeutic index, dose margin, therapeutic efficacy, and organ toxicity. Long-lasting and target-specific nanoparticles are required to achieve these goals [31] [32]. Nevertheless one of the most difficult challenges in cancer treatment, where the tumour can manifest as distinct metastases in various organs [33]. The properties of nanoparticles and their applications are summarized in Table 1.

\subsection{Photo-Thermal}

In this approach, nanoparticles are delivered to a specific region of interest (ROI) within the human body that has been exposed using microwave or laser, where cancer cells are found. For example, by administering nanoparticles, such as gold nanoparticles (Au-NPs) that respond to microwaves, the temperature within ROI can be raised by converting light energy exposure into heat induced by the nanoparticles [34] [35], though, in this manner, localized hyperthermia is achieved. Furthermore, another nanostructure has been progressed carbon-based graphene that are alert to laser beam such as carbon nanotube [36] [37], fullerene [38] [39], and graphene [40]. It is worth noting that gold nanoparticles are widely used due to gold's properties such as interactivity with light in the near-infrared (NIR) region $(700 \mathrm{~nm}-850 \mathrm{~nm})$ and biological compatibility [41] (Figure 1). Furthermore, NIR between $700 \mathrm{~nm}$ and $850 \mathrm{~nm}$, known as the optical biological window, is the least absorbed by water, melanin, oxy-hemoglobin, and deoxy-hemoglobin. Several researchers have reported that varying the size, shape, and composition 
Table 1. Highlights some of the types of chemical structures nanoparticle and their applications in life sciences [31].

\begin{tabular}{|c|c|c|}
\hline Particle class & Materials & Applications \\
\hline Natural & Chitosan & Drug/Gene delivery \\
\hline Materials or & Dextrane & \\
\hline derivative & $\begin{array}{c}\text { Gelatine } \\
\text { Alginates } \\
\text { Liposomes } \\
\text { Starch }\end{array}$ & \\
\hline Dendrimers & Branched polymers & Drug delivery \\
\hline Fullerenes & Carbon based carriers & $\begin{array}{l}\text { Photodynamics } \\
\text { Drug delivery }\end{array}$ \\
\hline & $\begin{array}{c}\text { Polylactic acid } \\
\text { Poly(cyno)acrylates }\end{array}$ & \\
\hline Polymer carriers & $\begin{array}{l}\text { Polyethyleinemine } \\
\text { Block copolymers } \\
\text { Polycaprolactone }\end{array}$ & Drug/Gene delivery \\
\hline Ferrofluids & $\begin{array}{l}\text { SPIONS } \\
\text { USPIONS }\end{array}$ & Imagine (MRI) \\
\hline Quantum dots & $\mathrm{Cd} / \mathrm{Zn}$-selenides & $\begin{array}{c}\text { Imaging } \\
\text { In virto diagnostics }\end{array}$ \\
\hline Various & $\begin{array}{l}\text { Silca-nanoparticles } \\
\text { Mixture of above }\end{array}$ & Gene delivery \\
\hline
\end{tabular}

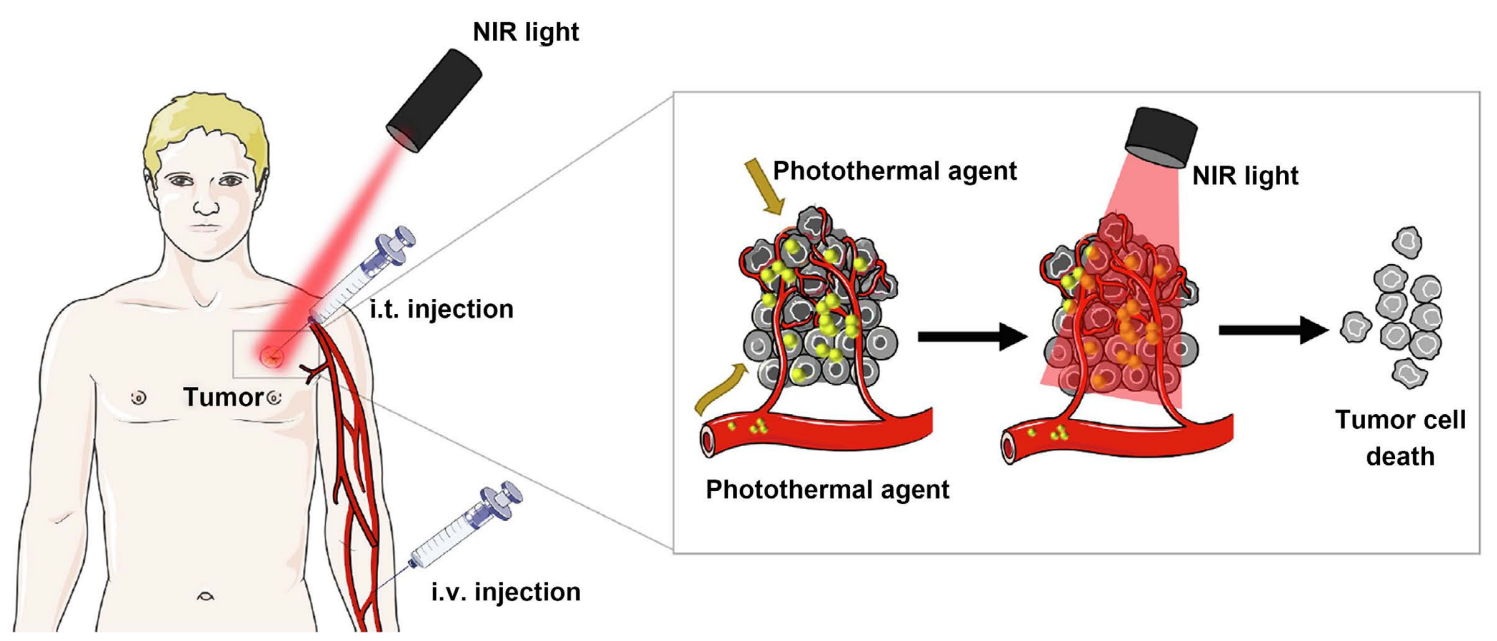

Figure 1. Depicts the PTT principle in cancer management. The photothermal agent is injected intravenously or intratumorally and accumulates in tumor tissue through the EPR effect or active cancer cell targeting. The tumor is then irradiated with NIR light at a specific power intensity for a short period of time (using either a continuous or a pulsed laser). The photothermal agent will then emit heat in response to NIR stimulation, resulting in local hyperthermia and tumor cell death [16].

of certain nanoparticles, such as gold, allows for tuning of the resonance wavelength interaction light [1] [42], core-shell gold nanoparticles [43] [44], hollow 
gold nanoshell [45] [46], gold nanorods [47], gold nanomatryoshkas [48], hybrid nanoshells [49] [50], and silver [51] [52].

\section{Theoretical Model}

Surface plasmon resonance is defined as the resonant oscillation of a free electron on the particle surface caused by an incident photon beam (SPR) [53]. Extensively, SPR occurs when the particle's size becomes comparable to the wavelength of the external light source. This notable property can be found in Mie theory's expression of the extinction cross section of a metal sphere at the dipole electric order [54]. Electrons in nanoparticles absorb electromagnetic energy (photons) and become excited to a higher energy state in this process, which is also known as nano-photosensitizers. The absorbed electromagnetic energy is converted to heat spread in the lattice during the electron-phonon relaxation process [55]. The heat distribution of electron-phonon scattering inside a spherical particle in a quasi-stationary environment is approximated by [56] [57] [58]:

$$
\tau_{r} \sim \frac{d^{2}}{16 \chi}
$$

where $d$ is the size of nanoparticles (diameter), $\chi$ is the thermal diffusivity of the nanoparticle. In case of a short pulse, two cases may occur: if pulse duration $\left(t_{p}\right)$ is less than $\tau_{r}$ there will be no heat exchanged between the nanoparticle and the surrounding medium, where heat is said to be confined within the nanoparticle. On the other hand, an exchange occurs if $t_{p}$ is greater than $\tau_{r}$ by the process of diffusion [59] [60]. $\mathrm{TiO}_{2}$ nanoparticles, for example, are eliminated by light at a specific wavelength in the process of photosensitizing, a molecule that produces a chemical change in another molecule in a photochemical process. Photo-induced electrons and holes can then react with hydroxyl ions or water to form oxidative radicals such as reactive oxygen species (ROS) and singlet oxygen. These species' generation is lethal to cancerous cells. Photo-thermal therapy employs a near infrared (NIR) light source to irradiate cancerous cells because it has a greater penetration capability in the human body than visible light [61]. It has been claimed that the exchange heat between the nanoparticle and the surrounding can be satisfied by using continuous wave (CW) that is determined by using the following equation [62] [63]

$$
\Delta T=T_{r}-T_{p}=\frac{Q_{p}}{4 \pi k r}
$$

where $T_{p}$ is the initial temperature of both the nanoparticle and the surrounding tissue, $Q_{p}$ is the heat produced by the nanoparticle, and $k$ is the thermal conductivity of the medium. The heat produced by the nanoparticle $\left(Q_{p}\right)$ can be estimated as

$$
Q_{p}=C_{a b s} I
$$

where $I$ is the fluency of the light source, and $C_{a b s}$ is the absorption coefficient of the cross-sectional area and is equal to (geometrical cross-sectional, $\sigma$ ) multip- 
lied by the absorption coefficient $\left(Q_{p}\right), C_{a b s}=\sigma Q_{a b s}$, Hence, the specific absorption rate $(S A R)$ can be determined through each particle exposed to laser fluency [64]

$$
\operatorname{SAR}\left[\mathrm{W} / \mathrm{m}^{2}\right]=N Q_{p}=N C_{a b s} I
$$

count on the laser fluency, the heat for the targeted tissue, including various thermodynamic and thermo-biological, is incrementally increased.

\subsection{Magnetic Nanoparticles Hyperthermia}

A key aspect of researching an alternative approach to conventional hyperthermia is the use of a magnetic field in situations where the heat distribution profile is inhomogeneous (Figure 2). This could cause heat to build up in collateral tissues, causing damage to healthy cells. In the same context, this inhomogeneous heat may miss the core of the cancerous cells, allowing it to worsen in the long run [62] [63]. The mechanism of using magnetic materials in hyperthermia is as follows: after saturation of ferromagnetic materials with a magnetic field, all magnetic domains align in the direction of the applied magnetic field. When the magnetic field is reversed, the magnetic domain aligns in the opposite direction. The hysteresis loop describes the process of varying the direction of the domain of the materials. The sizes of these ferromagnetic materials can be reduced to tiny nanoparticles, and varying their magnetization direction back and forth, randomly and rapidly, corresponds to temperature changes. Notable examples include superparamagnetic nanoparticles, which lose their magnetization in the absence of an external magnetic field, resulting in an efficient heat generator. These superparamagnetic nanoparticles, for example, behave like a paramagnet [1]. In recent years, there has been a surge of interest in superparamagnetic iron

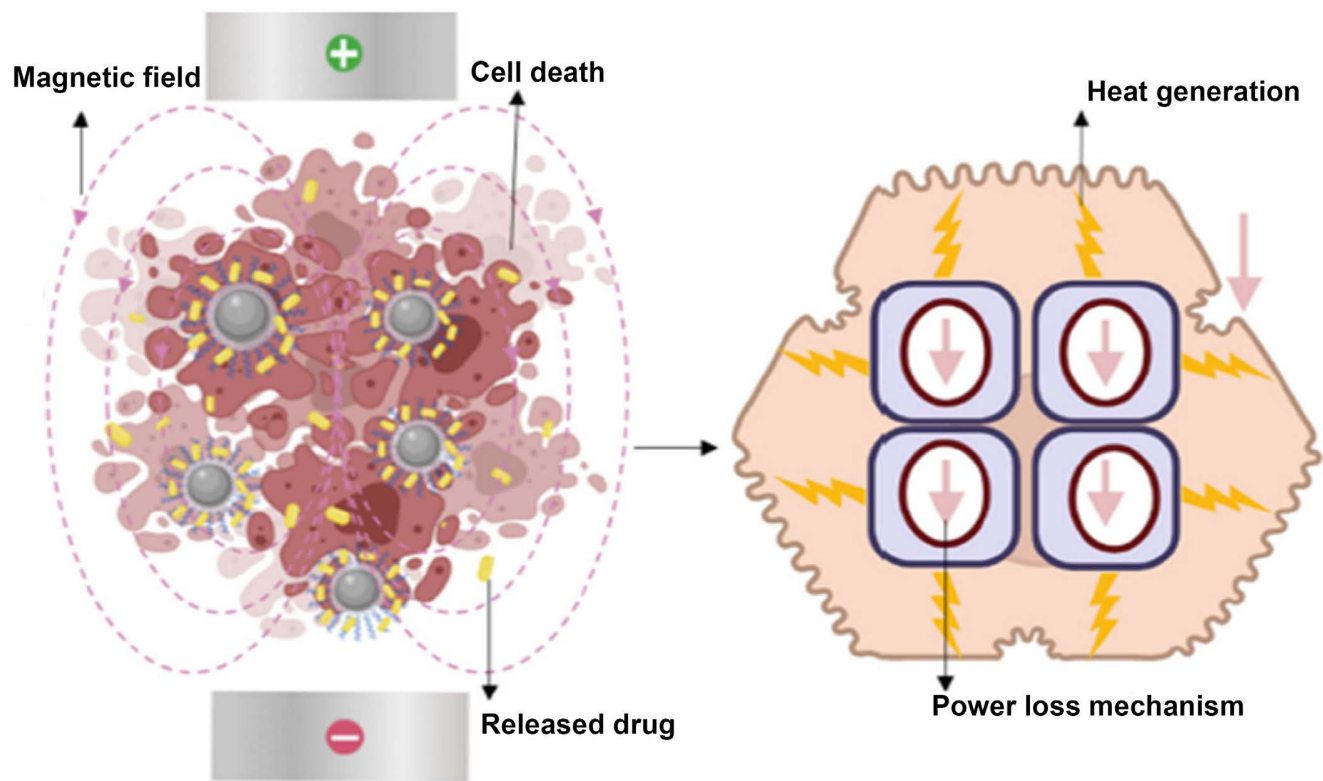

Figure 2. Shows MNPs being injected and then exposed to AMF to generate the desired heat in the tumor as the target inside the body [65]. 
oxide nanoparticle (SPION), a subset of iron oxide nanoparticle [64]. SPION has a significantly higher susceptibility than paramagnetic material, causing AMF to rapidly flip its polarity, resulting in heat generation due to hysteretic loss.

\section{Theoretical Model}

In general, most nanoparticle injection delivery methods in local hyperthermia treatment involve coating the magnetic nanoparticles with a tumor-specific antibody and injecting it into the bloodstream. The nanoparticles are then exposed to energy produced by various techniques such as radio frequency, microwave, and ultrasound. There are four methods available: arterial injection, direct injection, in situ implant formation, and active targeting [66] [67]. Arterial injection is a technique in which a fluid containing magnetic nanoparticles is injected into the bloodstream and then drains into the tumor. The most common method is direct injection, in which the fluid containing the nanoparticles is injected into the tumor. In situ implant formation method, injectable formulations that form gels as hydrogels (such as chitosan and sodium alginate) and organogels (such as ethylene-co-vinyl alcohol and cellulose acetate) to entangle magnetic particles in the tumor location.

Conceptually, in hyperthermia technology, within the tumour location, the electromagnetic is converted into heat by utilizing an external alternative force of magnetic field. Linear response theory has been used [68] to interpret the heat generated where the power $P$ generated per unit mass of particles exposed to a magnetic field with an amplitude $H_{0}$ and frequency $f$ can be written as:

$$
P=\pi \mu_{0} H_{0}^{2} f \chi_{0} \frac{2 \pi f \tau}{1+2 \pi f \tau}
$$

where $\mu_{0}$ is the permeability of magnetic field in free space and $\chi_{0}$ is the susceptibility, which estimates the ability of a material to become magnetic when exposed to a magnetic field, as the $\chi_{0}$ expressed as:

$$
\chi_{0}=\frac{\mu_{0} M_{s}^{2} V_{p}}{3 \rho k T} \frac{3}{\xi}\left(\operatorname{coth}(\xi)-\frac{1}{\xi}\right) ; \xi=\frac{\mu_{0} M_{s} V_{p} H_{0}}{k T}
$$

when applying the alternating magnetic field, the magnetic moments of the nanoparticles change their orientation according to the magnetic field direction. For relaxation of the particles' moments to its equilibrium, state causes energy dissipation as heat by two main mechanisms: Brownian rotation and Néel relaxation [69] [70]. Brownian rotation is dominant for the particles above a certain threshold and occurs in a magnetic fluid due to the viscosity of the carrier liquid $(\eta)$. Brownian rotation can be expressed as:

$$
\tau_{B}=\frac{3 \eta V_{H}}{k T}
$$

where $\tau_{B}$ is the Brownian relaxation time, $\eta$ is the viscosity, $V_{H}$ is the hydro-dynamic volume of the particle, $k$ is the Boltzmann constant and $T$ is temperature. Néel relaxation is a result of random flips of the spin without rotation of the particle, which is the only relaxation in the zero magnetic field within the 
tumour site, can be written as:

$$
\tau_{N}=\frac{\sqrt{\pi}}{2} \tau_{0} \frac{\mathrm{e}^{\sigma}}{\sigma}
$$

where $\sigma=\frac{K V_{M}}{K T}$ such that $\tau_{N}$ is the Néel relaxation time, $\tau_{0}$ is the characteristic relaxation time that is assumed to be equaled to $10^{-9}$ seconds, $K$ is anisotropy constant and $V_{M}$ is the volume of the particle. In the case where both relaxations happen simultaneously, the effective relaxation time can be expressed as:

$$
\tau=\frac{\tau_{B} \tau_{N}}{\tau_{B}+\tau_{N}}
$$

such that $\tau$ is the relaxation time for the simultaneous occurring of both relaxation events, where the size of the particle directly correlates to the Brownian. The heat generated by magnetic nanoparticles is quantified in term of the specific absorption rate $(S A R)$ equation that can be modelled as:

$$
S A R=C \frac{\Delta T}{\Delta t}
$$

where $C$ is the specific heat capacity of the sample and $\frac{\Delta T}{\Delta t}$ is the rising temperature [71].

\section{Bio-Heat Equation}

It is critical to predict the transient temperature distribution of the target tissues in advance to ensure optimal clinical outputs for biomedical applications. In a tumor hyperthermia process, for example, the primary goal is to raise the temperature of the diseased tissue to a therapeutic value, typically above $43^{\circ} \mathrm{C}$, and then thermally destroy it [72]. A thorough understanding of the extent of the irregular shape of the frozen region, the direction of ice growth, and the temperature distribution within the ice balls during the freezing process is a must for clinical operations to be successful. As a result, resolving bioheat transfer issues is critical for both hyperthermia and cryosurgery. Furthermore, similar bioheat transfer issues are frequently encountered in thermal diagnostics, thermal comfort analysis, brain hypothermia resuscitation, and burn injury evaluation [73].

Theoretical background used to study the profile of the heat in hyperthermia is the bioheat equation proposed by Pennes [74] that can be written as:

$$
\rho c \frac{\partial T(X, t)}{\partial t}=\nabla \cdot k(X) \nabla T(X, t)+Q_{b}(X, t)+Q_{m}(X, t)+Q_{r}(X, t)
$$

where $\rho$ and $c$ are the density and the specific heat of tissue, respectively; $\rho_{b}$ and $c_{b}$ denote the density and the specific heat of blood, respectively; $X$ contains the Cartesian coordinates $x, y$ and $z, k(X)$ is the space dependent thermal conductivity; $Q_{m}(X, t)$ is the metabolic heat generation; and $Q_{r}(X, t)$ denotes the distributed volumetric heat source due to an externally applied spatial heat- 
ing. $Q_{b}(X, t)$ is the blood heat generation, i.e. the heat generation within the environment; it is expressed as:

$$
Q_{b}(X, t)=\rho_{b} c_{b} \omega_{b}(X)\left[T_{a}-T(X, t)\right]
$$

where $\rho_{b}$ and $c_{b}$ correspond to the density and the specific heat of blood respectively; $\omega_{b}(X)$ is the space dependent blood perfusion, which is the value of blood perfusion that indicates the blood flow rate per unit tissue volume. This is mainly attributed to the microcirculation of the capillary network along with small arterioles and venules. $T_{a}$ is the blood temperature in the arteries supplying the tissue and is often regarded as a constant at $37^{\circ} \mathrm{C} ; T(X, t)$ is the tissue temperature. The solutions to bioheat transfer problems are of great significance in a wide variety of biomedical applications [75]. Especially, understanding the distribution in biological tissues involving either increasing or decreasing the temperature for many clinical practices such as hyperthermia [76].

\section{Conclusions}

Overall, hyperthermia treatment is a non-invasive process that raises tumor temperature to increase blood flow, oxygenation, and make tumor cells more sensitive to radiation. Radiation oncologists can improve tumor control while minimizing heat damage to healthy tissue by incorporating hyperthermia into radiation therapy. Many processes, such as laser, ionizing radiation, microwave, and alternating magnetic field, have been implemented. Although these processes allow for an increase in temperature within the targeted tissue, they also have side effects on nearby healthy tissue. Ionizing radiation, for example, may cause genetic mutation, and microwave may fail to target a specific tissue. Another approach is to damage, kill, or make cancer cells more sensitive to radiation effects within the targeted tissue while leaving healthy tissue alone. In medical oncology, nanotechnology offers a novel tool in the form of hyperthermia, which allows for the remote induction of local heat. One prominent example is the ability of some magnetic nanoparticles to convert electromagnetic energy into heat in targeted tissues where tumor cells and nanoparticles are combined in the same location. As a result, the temperature can be raised locally to a level greater than $40^{\circ} \mathrm{C}$, inflicting sufficient damage and possibly killing cancer cells. Furthermore, rising temperatures may make cancer cells more susceptible to the effects of radiation and certain anti-cancer drugs. In this case, a nanoparticle serves as a tiny heater that can be controlled externally by using electromagnetic radiation generated by an alternating magnetic field at frequencies ranging from a few $\mathrm{kHz}$ to $1 \mathrm{MHz}$. This range of electromagnetic radiation is safe for human tissue and has the ability to penetrate deeper organs or tissue. It is worth noting that these tumor-specific nanoparticle localization techniques produce tumor-specific dose delivery that is independent of the invasive procedures used in other localized dose-delivery strategies, such as interstitial hyperthermia. Targeted nanoparticles also cause less nonspecific accumulation in the body, allowing for a lower nanoparticle injection dose to achieve the same therapeutic effect 
and lowering the risk of nanoparticle-mediated toxicity. Several challenges confront the use of nanoparticles in hyperthermia, including: adequacy and uniformity of nanoparticle accumulation at the tumor site, quality control issues impeding clinical translation of nanoparticles, biocompatibility related to toxicity, and delaying effects of retained nanoparticles. One of the most difficult challenges in drug delivery is getting the drug to where it is needed in the body while avoiding potential side effects to non-diseased organs. This is especially difficult in cancer treatment, where the tumor can manifest as distinct metastases in various organs.

A simulation model based on solving the bio-heat equation with certain conditions was used to optimize the amount of heat required to kill the cancer cells. In hyperthermia, where the magnetization becomes zero after removing the alternating magnetic field, superparamagnetic, single domain nanoparticles are typically recommended.

\section{Conflicts of Interest}

The author declares no conflicts of interest regarding the publication of this paper.

\section{References}

[1] Kaur, P., Aliru, M.L., Chadha, A.S. and Asea, A. (2016) Hyperthermia Using Nanoparticles-Promises and Pitfalls. International Journal of Hyperthermia, 32, 1-24. https://doi.org/10.3109/02656736.2015.1120889

[2] Zhao, G., Wang, L., Cheng, R., Mao, L., Arnold, R., Howerth, E., Chen, Z. and Platt, S. (2012) Magnetic Nanoparticle-Based Hyperthermia for Head \& Neck Cancer in Mouse Model. Theranostics, 2, 113-121. https://doi.org/10.7150/thno.3854

[3] Beik, J., Abed, Z., Ghoreishi, F., Hosseini-Nami, S., Mehzadi, S., Shakri-Zadeh, A. and Kamrava, S. (2016) Nanotechnology in Hyperthermia Cancer Therapy: From Fundamental Principles to Advanced Applications. Journal of Controlled Released, 235, 206-221. https://doi.org/10.1016/j.jconrel.2016.05.062

[4] Sneed, P., Stauffer, P. and Stege, G. (2004) Hyperthermia. In: Leibel, S.A. and Phillips, T.L., Eds., Textbook of Radiation Oncology, 2nd Edition, Demos Medical Publishing, New York, 1569-1596.

[5] Roussakow, S. (2013) The History of Hyperthermia Rise and Decline. Conference Papers in Science, 2013, Article ID: 428027. https://doi.org/10.1155/2013/428027

[6] van der Zee, J. (2002) Heating the Patient: A Promising Approach? Annals of Oncology, 13, 1173-1184. https://doi.org/10.1093/annonc/mdf280

[7] Chatterjee, D.K., Diagaradjane, P. and Krishnan, S. (2011) Nanoparticle-Mediated Hyperthermia in Cancer Therapy. Therapeutic Delivery, 2, 1001-1014. https://doi.org/10.4155/tde.11.72

[8] Banobre-Lopez, M., Tiejeiro, A. and Rivas, J. (2013) Magnetic Nanoparticle-Based Hyperthermia for Cancer Treatment. Reports of Practical Oncology and Radiotherapy, 18, 397-400. https://doi.org/10.1016/j.rpor.2013.09.011

[9] Wust, P., Hildebrandt, B., Sreenivasa, G., et al. (2002) Hyperthermia in Combined Treatment of Cancer. The Lancet Oncology, 3, 487-497. https://doi.org/10.1016/S1470-2045(02)00818-5 
[10] Hildebrandt, B., Wust, P., Ahlers, O., Dieing, A., Sreenivasa, G., Kerner, T., Felix, R. and Riess, H. (2002) The Cellular and Molecular Basis of Hyperthermia. Critical Reviews in Oncology/ Hematology, 43, 33-65. https://doi.org/10.1016/S1040-8428(01)00179-2

[11] Yang, K.-L., Huang, C.-C., Chi, M.-S., Chiang, H.-C., Wang, Y.-S., Hsia, C.-C., Andocs, G., Wang, H.-E. and Chi, K.-H. (2016) In Vitro Comparison of Conventional Hyperthermia and Modulated Electro-Hyperthermia. Oncotarget, 7, 84082-84092. https://doi.org/10.18632/oncotarget.11444

[12] Hartung, G. and Mansoori, G.A. (2013) In Vivo General Trends, Filtration and Toxicity of Nanoparticles. Journal of Nanomaterials \& Molecular Nanotechnology, 2, 1-21.

[13] Keyhanian, K., Mansoori, G.A. and Rahimpour, M. (2010) Prospects for Cancer Nanotechnology Treatment by Azurin. Dynamic Biochemistry, Process Biotechnology and Molecular Biology, 4, 48-66.

[14] Khoei, S., Mahdavi, S.R., Fakhimikabir, H., Shakeri-Zadeh, A. and Hashemian, A. (2014) The Role of Iron Oxide Nanoparticles in the Radiosensitization of Human Prostate Carcinoma Cell Line DU145 at Megavoltage Radiation Energies. International Journal of Radiation Biology, 90, 351-356. https://doi.org/10.3109/09553002.2014.888104

[15] Shah, A. and Dobrovolskaia, M.A. (2018) Immunological Effects of Iron Oxide Nanoparticles and Iron-Based Complex Drug Formulations: Therapeutic Benefits, Toxicity, Mechanistic Insights, and Translational Considerations. Nanomedicine and Nanotechnology, 14, 977-990. https://doi.org/10.1016/j.nano.2018.01.014

[16] Granja, A., Pinhriro, M., Sousa, C.T. and Reis, S. (2021) Gold Nanostructures as Mediators of Hyperthermia Therapies in Breast Cancer. Biochemical Pharmacology, 190, Article ID: 114639. https://doi.org/10.1016/j.bcp.2021.114639

[17] Dewhirst, M.W., Gibbs, F.A.J., Roemer, R.B. and Samulski, T.V. (2000) Clinical Radiation Oncology. Churchill Livingstone, New York, 256-282.

[18] Horowitz, L.F., Rodriguez, Z.D.-K., Lin, R., Castro, K., Mikheev, A.M., et al. (2020) Multiplexed Drug Testing of Tumor Slices Using a Microfluidic Platform. Precision Oncology, 4, 1-12. https://doi.org/10.1038/s41698-020-0117-y

[19] Feldman, A.L., Libutti, S.K., Pingpank, J.F., Bartlett, D.L., Beresnev, T.H., Mavroukakis, S.M., et al. (2003) Analysis of Factors Associated with Outcome in Patients with Malignant Peritoneal Mesothelioma Undergoing Surgical Debulking and Intraperitoneal Chemotherapy. Journal of Clinical Oncology, 21, 4560-4567. https://doi.org/10.1200/JCO.2003.04.150

[20] Seegenscumied, M.H., Fessenden, P. and Vernon, C.C. (1995) Thermoradiotherapy and Thermochemotherapy. Vol. 1, Biology, Physiology and Physics. Springer-Verlag, Berlin. https://doi.org/10.1007/978-3-642-57858-8

[21] Geelen, W.P., Hesse, U., Hemptinne, B. and Pattyn, P. (2000) Hyperthermic Intraperitoneal Chemoperfusion in the Treatment of Locally Advanced Intraabdominal Cancer. British Journal of Surgery, 87, 1006-1015. https://doi.org/10.1046/j.1365-2168.2000.01538.x

[22] Wehner, H., Von Ardenne, A. and Kaltofen, S. (2001) Whole Body Hyperthermia with Water-Filtered Infrared Radiation: Technical-Physical Aspects and Clinical Experiences. International Journal of Hyperthermia, 17, 19-30. https://doi.org/10.1080/02656730150201561

[23] Punit, K., Aliru, M.L., Chadha, A.S., Asea, A. and Krishnan, S. (2016) Hyperthermia Using Nanoparticles-Promises and Pitfalls. International Journal of Hyperthermia, 
32, 76-88. https://doi.org/10.3109/02656736.2015.1120889

[24] Singer, A., Markousta, E., Limayem, A., Subhra, M. and Mohapartra, S.S. (2018) Nano Biotechnology Medical Applications: Overcoming Challenges through Innovation. Medicine and Biotechnology, 2, 146-160. https://doi.org/10.2478/ebtj-2018-0019

[25] Liu, Y., Zhang, P., Jin, X., Li, J., Chen, W. and Li, Q. (2018) Metal-Based Nano-Enhancers for Future Radiotherapy: Radiosensitizating and Synergetics Effects on Tumor Cells. Theranostics, 8, 1824-1849. https://doi.org/10.7150/thno.22172

[26] Burda, C., Chen, X., Narayanan, R. and El-Sayed, A.M. (2005) Chemistry and Properties of Nanocrystals of Different Shapes. Chemical Reviews, 105, 1025-1102.

https://doi.org/10.1021/cr030063a

[27] Schwartz, J.A., Price, R.E., Gill-Sharp, K.L., Sang, K.L., Khorchani, J., Goodwin, B.S. and Payne, J.D. (2011) Selective Nanoparticle-Directed Ablation of the Canine Prostate. Lasers in Surgery and Medicine, 43, 213-220. https://doi.org/10.1002/1sm.21039

[28] Mocan, L., Metea, C., Tabaran, F.A. and Mosteanu, O. (2017) Selective ex Vivo Photothermal Nano-Therapy of Solid Liver Tumors Mediated by Albumin Conjugated Gold Nanoparticles. Biomaterials, 119, 33-34. https://doi.org/10.1016/j.biomaterials.2016.12.009

[29] Hejmady, S., Pradhan, R., Alexander, A., Agrwal, M., Singhvi, G., et al. (2020) Recent Advances in Targeted Nanomedicine as Promising Antitumor Therapeutics. Drug Discovery Today, 25, 2227-2244. https://doi.org/10.1016/j.drudis.2020.09.031

[30] Zhao, L., Zhang, X., Wang, X., Guan, X., Zhang, W. and Ma, J. (2021) Recent Advances in Selective Photothermal Therapy of Tumor. Journal of Nanobiotechnolo$g y$, 19, 2-15. https://doi.org/10.1186/s12951-021-01080-3

[31] Jong, W. and Borm, P. (2008) Drug Delivery and Nanoparticles: Applications and Hazards. International Journal of Nanomedicine, 3, 133-149.

https://doi.org/10.2147/IJN.S596

[32] Khan, I., Saeed, K. and Khan, I. (2019) Nanoparticles: Properties, Applications and Toxicities. Arabian Journal of Chemistry, 12, 908-931. https://doi.org/10.1016/j.arabjc.2017.05.011

[33] Kerbag, Y., Betrouni, N., Collinet, P., Hzais, H., Mordon, S., Dewella-Vignion, A.-S. and Merlot, B. (2017) Laser Interstitial Thermotherapy Application for Breast Surgery: Current Situation and New Trends. The Breast, 33, 145-152. https://doi.org/10.1016/j.breast.2017.03.016

[34] Nikolaos, M.D., Pavlopoulou, A., Tremi, I., Kouloulias, V., Georgios, T. and Georgakilas, A.G. (2019) Prediction of Gold Nanoparticle and Microwave-Induced Hyperthermia Effests on Tumor Conterol via a Simulation Approach. Nanomaterials, 9, 1-19. https://doi.org/10.3390/nano9020167

[35] Chichet, A., Skowronek, J., Kubaszewska, M. and Kanikowski, M. (2007) Hyperthermia-Description of a Method and a Review of a Clinical Applications. Reports of Practical Oncology and Radiotherapy, 12, 267-275. https://doi.org/10.1016/S1507-1367(10)60065-X

[36] Zhou, F., Xing, D., Ou, Z., Wu, B., Resasco, D.E. and Chen, W.R. (2009) Cancer Photothermal Therapy in the Near-Infrared Region by Using Single-Walled Carbon Nanotubes. Journal of Biomedical Optics, 14, Article ID: 021009. https://doi.org/10.1117/1.3078803

[37] Cui, X. (2019) Self-Thermoregulating Ferromagnetic FeNi Filled Carbon Nanotubes for Magnetic Hyperthermia Cancer Therapy. IOP Conference Series: Earth and En- 
vironmental Science, 310, Article ID: 042016. https://doi.org/10.1088/1755-1315/310/4/042016

[38] Zaibaq, N.G., Pollard, A.C., Collins, M.J., Pisaneschi, F., Pagel, M.D. and Wilson, L.J. (2020) Evaluation of the Biodistribution of Serinolamide-Derivatized C60 Fullerene. Nanomaterials, 10, 143. https://doi.org/10.3390/nano10010143

[39] Nalepa, P., Geweki, R., Szewczyk, G., et al. (2020) A [60]fullerene Nanoconjugate with Gemcitabine: Synthesis, Biophysical Properties and Biological Evaluation for Treating Pancreatic Cancer. Cancer Nano, 11, 1-22. https://doi.org/10.1186/s12645-020-00058-4

[40] Miyazaki, T., Akaike, J., Kawashita, M. and Lim, H.N. (2019) In Vitro Apatite Mineralization and Heat Generation of Magnetite-Reduced Graphene Oxide Nanocomposites for Hyperthermia Treatment. Materials Science and Engineering. C, 99, 68-72. https://doi.org/10.1016/j.msec.2019.01.091

[41] Kennedy, L.C., Bickford, L.R., Lewinski, N.A., et al. (2011) A New Era for Cancer Treatment: Gold-Nanoparticle-Mediated Thermal Therapies. Small, 7, 169-183. https://doi.org/10.1002/smll.201000134

[42] Alghlayini, A., Jiang, L., Gu, X., Yeoh, G.H., Cranfield, C.G., Timchenko, V., Cornell, B.A. and Valezuela, S.M. (2020) Transfer between Gold Nanoparticles and Tethered Bilayer Lipid Membranes. BBA-Biomembranes, 1862, Article ID: 183334. https://doi.org/10.1016/j.bbamem.2020.183334

[43] O’Neal, D.P., Hirsch, L.R., Halas, N.J., Payne, J.D. and West, J.L. (2004) Photo-Thermal Tumor Ablation in Mice Using near Infrared-Absorbing Nanoparticles. Cancer Letters, 209, 171-176. https://doi.org/10.1016/j.canlet.2004.02.004

[44] Atinson, R.L., Zhang, M., Diagaradjane, P., Piddibhotla, S., Contreras, A. and Hilsenbeck, S.G. (2010) Thermal Enhancement with Optically Activated Gold Nanoshells. Science Translational Medicine, 2, 55 ra79. https://doi.org/10.1126/scitranslmed.3001447

[45] Goodman, A.M., Cao, Y., Urban, C., Neumann, O., Ayala-Orozco, C., Knight, M.W., et al. (2014) The Surprising in Vivo Instability of Near-IR-Absorbing Hollow Au-Ag Nanoshells. Acs Nano, 8, 3222-3231. https://doi.org/10.1021/nn405663h

[46] Matlashov, M.E., Scherbbakova, D.M., Alvelid, J., et al. (2020) A Set of Monomeric Near-Infrared Fluorescent Proteins for Multicolor Imaging across Scales. Nature Communications, 11, Article No. 239. https://doi.org/10.1038/s41467-019-13897-6

[47] Puleio, R., et al. (2020) Effect of Actively Targeted Copolymer Coating on Solid Tumors Eradication by Gold Nanorods-Induced Hyperthermia. International Journal of Pharmaceutics, 587, 119641-119646.

https://doi.org/10.1016/j.ijpharm.2020.119641

[48] Ayala-Orozco, C., Urban, C., Knights, M.W., Urban, A.S., Neumann, O., Bishnoi, S.W., et al. (2014) Sub-100 nm Gold Nanomatryoshkas Improve Photo-Thermal Therapy Efficacy in Large and Highly Aggressive Triple Negative Breast Tumors. Journal of Controlled Release, 191, 90-97. https://doi.org/10.1016/j.jconrel.2014.07.038

[49] Nunes, T., Pons, T., Hou, X., et al. (2019) Pulsed-Laser Irradiation of Multifunctional Gold Nanoshells to Overcome Trastuzumab Resistance in HER2-Overexpressing Breast Cancer. Journal of Experimental \& Clinical Cancer Research, 38, Article No. 306. https://doi.org/10.1186/s13046-019-1305-X

[50] Li, Y., Dhawan, U., Wang, H.-Y., Liu, X., Ku, H.-H., Tsai, M.-T., Yen, H.-W. and Chang, R.-J. (2019) Theranostic Iron@Gold Core-Shell Nanoparticles for Simultaneous Hyperthermia-Chemotherapy upon Photo-Stimulation. Particle \& Particle 
Systems Characterization, 36, Article ID: 1800419. https://doi.org/10.1002/ppsc.201800419

[51] Zakomirnyi, V.I., Rinkevicius, Z., Baryshinkov, G.V., Sorensen, L.K. and Agren, H. (2019) Extended Discrete Interaction Model: Plasmonic Excitations of Silver Nanoparticles. The Journal of Physical Chemistry C, 123, 28867-28880.

https://doi.org/10.1021/acs.jpcc.9b07410

[52] Hepokur, C., Kariper, I.A., Misir, S., Ay, E., Tunoglu, S., Ersez, M.S., Zeybek, U., Kuruca, S.E. and Yaylim, I. (2019) Silver Nanoparticle/Capecitabine for Breast Cancer Cell Treatment. Toxicology in Vitro, 61, Article ID: 104600. https://doi.org/10.1016/j.tiv.2019.104600

[53] Zhao, S., Wu, S., Jia, Q., Huang, L., Lan, M. and Wang, P. (2020) Lysosome-Targetable Carbon Dots for Highly Efficient Photothermal/Photodynamic Synergistic Cancer Therapy and Photoacoustic/Two-Photon Excited Fluorescence Imaging. Chemical Engineering Journal, 388, Article ID: 124212.

https://doi.org/10.1016/j.cej.2020.124212

[54] Mie, G. (1908) Beiträge zur Optik trüber Medien, speziell kolloidaler Metallösungen. Annalen der Physik, 330, 377-445. https://doi.org/10.1002/andp.19083300302

[55] Lan, M., Zhao, S., Liu, W., Lee, C.-S., Zhang, W. and Wang, P. (2019) Photosensitizer for Photodynamic Therapy. Advanced Healthcare Materials, 8, Article ID: 1900132. https://doi.org/10.1002/adhm.201900132

[56] Gan, R., Fan, H., Wei, Z., Liu, H., Lan, S. and Dai, Q. (2019) Photothermal Response of Hollow Gold Nanorods under Femtosecond Laser Irradiation. Nanomaterials, 9, 711. https://doi.org/10.3390/nano9050711

[57] Raes, L., Hecke, C.V., Michiels, J., Stremersch, S., Fraire, J.C., et al. (2019) Gold Nanoparticle-Mediated Photoporation Enables Delivery of Macromolecules over a Wide Range of Molecular Weights in Human CD4+ T Cells. Crystals, 9, 411. https://doi.org/10.3390/cryst9080411

[58] Li, J., Xu, L., Lu, K., Shahzad, M.K. and Ren, J. (2019) Efficient Nanoheater Operated in a Biological. Biomedical Optics Express, 10, 1935-1941. https://doi.org/10.1364/BOE.10.001935

[59] Ren, Y., Qi, H., Chen, Q. and Ruan, L. (2017) Thermal Dosage Investigation for Optimal Temperature Distribution in Gold Nanoparticle Enhanced Photothermal Therapy. International Journal of Heat and Mass Transfer, 106, 212-221. https://doi.org/10.1016/j.ijheatmasstransfer.2016.10.067

[60] Kreith, F. and Black, W.Z. (1980) Basic Heat Transfer.

[61] McNamara, K. and Tofail, A.M. (2017) Nanoparticle in Biomedical Applications. Advances in Physics. X, 2, 54-88. https://doi.org/10.1080/23746149.2016.1254570

[62] Qin, Z. and Bischof, J.C. (2012) Thermophysical and Biological Responses of Gold Nanoparticle Laser Heating. Chemical Society Reviews, 41, 1191-1217. https://doi.org/10.1039/C1CS15184C

[63] Moros, E. (2012) Physics of Thermal Therapy: Fundamentals and Clinical Applications. CRC Press, Boca Raton.

[64] Reihemann, K., Schneeider, S.W., Luger, T.A., Godin, B., Ferrari, M. and Funchs, H. (2009) Nanomedicine-Challenge and Perspectives. Angewandte Chemie International Edition, 48, 872-897. https://doi.org/10.1002/anie.200802585

[65] Salmanian, G., Hassanzadeh-Tabrizi, S.A. and Koupaei, N. (2021) Magnetic Chitosan Nanocomposites for Simultaneous Hyperthermia and Drug Delivery Applications: A Review. International Journal of Biological Macromolecules, 184, 618-635. 
https://doi.org/10.1016/j.ijbiomac.2021.06.108

[66] Overchuka, M. and Gang, Z. (2017) Overcoming Obstacles in the Tumor Microenvironment: Recent Advancements in Nanoparticle Delivery for Cancer Theranostics. Biomaterials, 156, 217-237. https://doi.org/10.1016/j.biomaterials.2017.10.024

[67] Ouyang, B., et al. (2020) The Dose Threshold for Nanoparticle Tumour Delivery. Nature Materials, 19, 1362-1371. https://doi.org/10.1038/s41563-020-0755-Z

[68] Carriao, M.S., Aquino, V., Landi, G.T., Verde, E.L., Sousa, M.H. and Bakuszis, A.F. (2017) Giant-Spin Nonlinear Response Theory of Magnetic Nanoparticle. Journal of Applied Physics, 121, Article ID: 173901. https://doi.org/10.1063/1.4982357

[69] Ilg, P. (2019) Diffusion-Jump Model for the Combined Brownian and Néel Relaxation Dynamics of Ferrofluids in the Presence of External Fields and Flow. Physical Review E, 100, Article ID: 022608. https://doi.org/10.1103/PhysRevE.100.022608

[70] Ilg, P. and Kröger, M. (2020) Dynamics of Interacting Magnetic Nanoparticles: Effective Behavior from Competition between Brownian and Néel Relaxation. Physical Chemistry Chemical Physics, 22, 22244-22259.

https://doi.org/10.1039/D0CP04377I

[71] Bordet, A., Landis, R.F., Lee, Y., Tonga, G.Y., Asensio, J.M., Li, C.-H., Fazzini, P.-F., Soulantica, K., Rotello, V.M. and Chaudret, B. (2019) Water-Dispersible and Biocompatible Iron Carbide Nanoparticles with High Specific Absorption Rate. ACS Nano, 13, 2870-2878. https://doi.org/10.1021/acsnano.8b05671

[72] Roemer, R.B. (1999) Engineering Aspects of Hyperthermia Therapy. Annual Review of Biomedical Engineering, 1, 347-376.

https://doi.org/10.1146/annurev.bioeng.1.1.347

[73] Deng, Z.S. and Liu, J. (2002) Analytical Study on Bioheat Transfer Problems with Spatial or Transient Heating on Skin Surface or Inside Biological Bodies. ASME Journal of Biomechanical Engineering, 124, 638-649. https://doi.org/10.1115/1.1516810

[74] Pennes, H.H. (1948) Analysis of Tissue and Arterial Blood Temperatures in the Resting Human Forearm. Journal of Applied Physiology, 1, 93-122. https://doi.org/10.1152/jappl.1948.1.2.93

[75] Liu, J. and Wang, C.C. (1997) Bioheat Transfer. Science Press, Beijing, 435 p. (In Chinese)

[76] Liu, J. and Deng, Z.S. (2008) Physics of Tumor Hyperthermia. Science, Beijing. (In Chinese) 